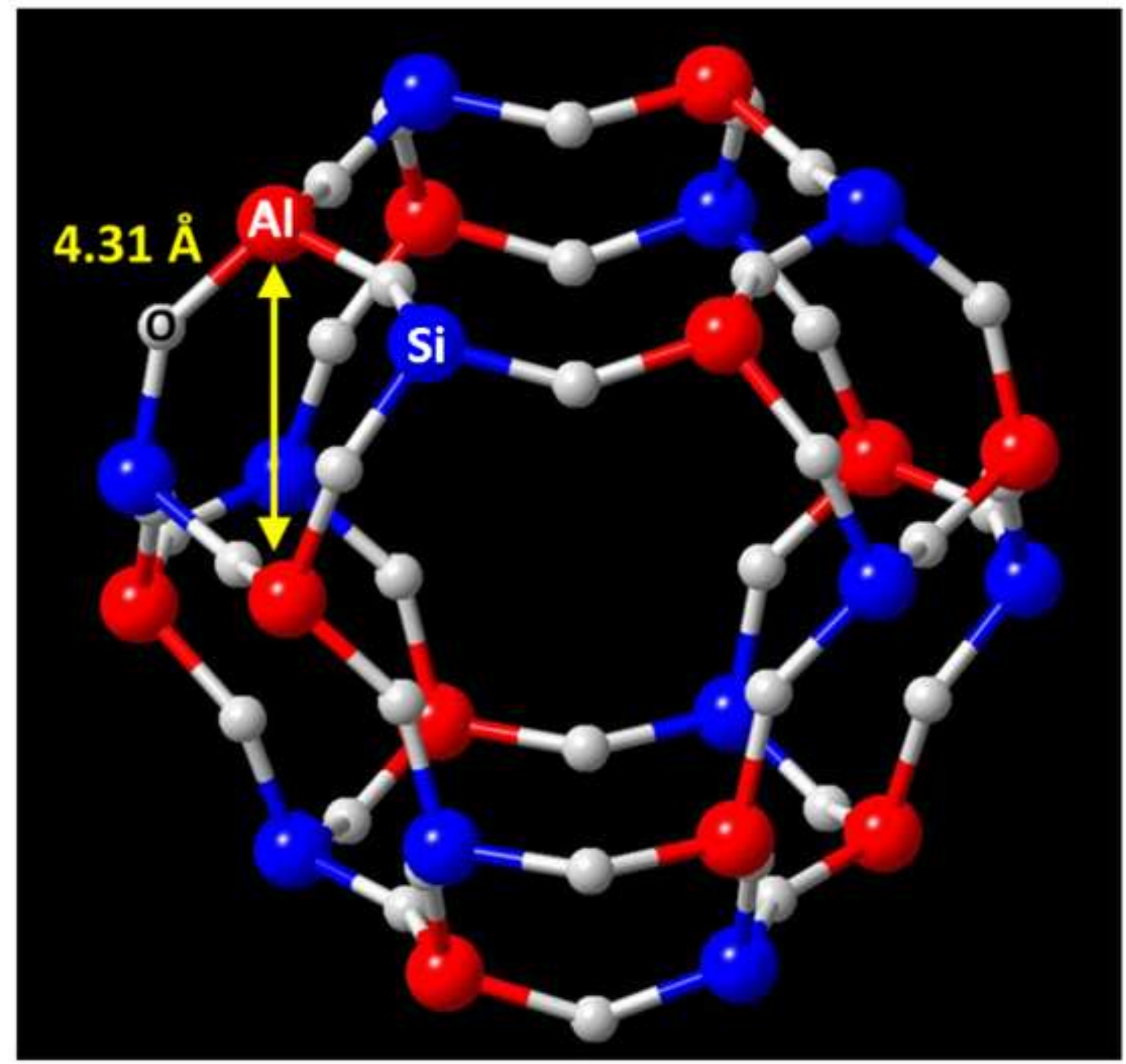




\title{
Imaging individual lanthanum atoms in zeolite $Y$ by scanning transmission electron microscopy: evidence of lanthanum pair sites
}

\author{
Pinghong Xu, ${ }^{1}$ Jing Lu, ${ }^{1}$ Ceren Aydin, ${ }^{1}$ Louise Debefve, ${ }^{1}$ Nigel D. Browning, ${ }^{2}$ \\ Cong-Yan Chen, ${ }^{1,3^{*}}$ Bruce C. Gates ${ }^{1 *}$ \\ ${ }^{1}$ Department of Chemical Engineering and Materials Science, University of California, Davis, \\ CA 95616, USA \\ ${ }^{2}$ Fundamental and Computational Sciences Division, Pacific Northwest National Laboratory, \\ Richland, WA 99352, USA \\ ${ }^{3}$ Chevron Energy Technology Company, Richmond, CA 94802, USA \\ *e-mail: \\ cychen@,chevron.com (C. Y. Chen) \\ bcgates@ucdavis.edu (B. C. Gates).
}




\begin{abstract}
Images of La-exchanged $\mathrm{NaY}$ zeolite obtained with aberration-corrected scanning transmission electron microscopy (STEM) show that about $80 \%$ of the La cations were present as site-isolated species, with the remainder in pair sites. The distance between La cations in the pair sites ranged from 1.44 to $3.84 \AA$, consistent with the presence of pairs of cations tilted at various angles with respect to the support surface. The actual distance between La cations in the pair sites is inferred to be approximately $3.84 \AA$. The results suggest the presence of dimeric structures of La cations bridged with $\mathrm{O}$ anions, and the presence of such species has been inferred previously on the basis of X-ray photoelectron spectroscopy (W. Grünert, U. Sauerlandt, R. Schlögl, H.G. Karge, J. Phys. Chem., 97 (1993) 1413).
\end{abstract}

Keywords: Lanthanum, zeolite Y, scanning transmission electron microscopy 


\section{Introduction}

Zeolites are used extensively as catalysts in petrochemical processing and petroleum refining, with catalytic cracking of petroleum being the largest-scale application [1-4]. Many commercial cracking catalysts incorporate faujasite-type zeolites that contain rare earth ions such as lanthanum, because they significantly improve the catalytic activity [5-7] and hydrothermal stability $[8,9]$ in many industrially relevant reactions. Characterizations of lanthanum-containing zeolites by X-ray diffraction crystallography, X-ray photoelectron spectroscopy (XPS), and infrared spectroscopy [10-12] provide details of the zeolite structure and demonstrate that $\mathrm{La}^{3+}$ ions hydrolyze water to form hydroxylated $\mathrm{La}^{2+}$ species. The stable crystallographic locations of the lanthanum ions vary with the treatment conditions. Before thermal treatment, the hydrated lanthanum cations are located in the type II and type V sites in the supercages of zeolite Y; thermal treatment at temperatures above $353 \mathrm{~K}$ partially removes water ligands, and lanthanum cations migrate through the 6-membered ring openings into the type I' positions in the sodalite cages $[13,14]$.

A point of debate about the lanthanum species in faujasite zeolites has to do with their possible bridging by hydroxyl groups (or oxygen); such bridged species have been postulated to be present in pairs, and XPS data have been interpreted as evidence confirming the presence of such pair sites [11], although some workers have expressed doubt about their presence on the basis of neutron diffraction data [15].

Our goal was to use electron microscopy to image lanthanum ions in zeolite $\mathrm{Y}$ and to seek evidence of lanthanum pair sites. This work was made possible by recent advances in aberration-corrected scanning transmission electron microscopy (STEM), which allows atomicresolution imaging of heavy metals in zeolites, and sometimes even identification of the 
locations of these atoms in the zeolite pore structure [16-18]. Imaging of zeolites with STEM is challenging because the electron beam readily damages the crystalline zeolite structure, and images must be obtained before such damage is detected. Now we report the first images showing individual La cations in a zeolite and demonstrate the presence of lanthanum pair sites.

\section{Experimental methods}

\subsection{Sample Synthesis}

$\mathrm{NaY}$ zeolite (Zeolyst $\mathrm{CBV} 100, \mathrm{Si} / \mathrm{Al}$ atomic ratio $\approx 2.5$ as stated by the supplier) was used as the starting material for the preparation of the lanthanum-exchanged Y zeolite (designated as LaNaY zeolite) as follows: NaY zeolite powder (2.5 g, as provided by the supplier) was slurried with $10 \mathrm{~mL}$ of $0.156-M$ aqueous $\mathrm{La}\left(\mathrm{NO}_{3}\right)_{3}$ at $353 \mathrm{~K}$ for ion exchange. The solution was replenished four times. Each of the first two exchanges took $90 \mathrm{~min}$, and the others took $180 \mathrm{~min}$ each. The zeolite was subsequently washed with de-ionized water, dried at $393 \mathrm{~K}$, and treated overnight under vacuum at $673 \mathrm{~K}$.

\subsection{Sample Characterization}

Standard X-ray diffraction, elemental analysis (Galbraith Laboratories, Knoxville, TN, USA), and liquid nitrogen adsorption methods were used to characterize both the NaY and LaNaY zeolite samples investigated in this work. STEM images of the LaNaY zeolite were obtained with a FEI Titan 80-300 electron microscope equipped with an FEG, operated at $300 \mathrm{kV}$, with a CEOS hexapole probe (STEM) aberration corrector. The images were captured with a high angle annular dark field (HAADF) detector with a collection semi-angle of 75-200 mrad and a probe convergence semi-angle of $17.5 \mathrm{mrad}$. A standard sample consisting of particles of gold on holey 
carbon (Ted Pella Supplies) was used to align the aberration corrector until atomic resolution was achieved, prior to recording of the images.

\section{Results}

The XRD data shown in Fig. 1a characterizing the parent NaY zeolite match expectations and show that the sample was highly crystalline. The XRD data presented in Fig. 1b characterizing the LaNaY zeolite indicate that the intensities of the peaks were generally decreased relative to those of the parent NaY zeolite shown in Fig. 1a, consistent with reported results $[9,19]$. The intensity of the first peak is considered to be 100 for normalization of the others. As is well known, such a decrease in the peak intensities is attributed, first, to the enhanced absorption of the incident X-rays by the heavy La cations, which affects all reflections - that is, all reflections including the normalized $100 \%$ reflection should become less intense. Moreover, lanthanum is a much stronger scatterer than sodium. After $\mathrm{Na}^{+}$is exchanged for $\mathrm{La}^{3+}$, the electron density distribution within a given unit cell is changed significantly, leading to different interference of the diffracted beam and thus different relative intensities of individual reflections. Therefore, the decrease in the peak intensities is inferred not to be indicative of a loss in crystallinity of the zeolite as a consequence of the lanthanum ion exchange; this inference is supported by the observation that the intensities of these XRD peaks were restored when LaNaY zeolite was exchanged back to NaY zeolite.

Elemental analysis showed that the composition of the LaNaY zeolite was $\mathrm{H}_{3.2} \mathrm{La}_{11.9} \mathrm{Na}_{16.5}\left(\mathrm{AlO}_{2}\right)_{55.4}\left(\mathrm{SiO}_{2}\right)_{136.6}\left(\mathrm{H}_{2} \mathrm{O}\right)_{254}$, indicating that approximately $65 \%$ of the $\mathrm{Na}^{+}$ions had been exchanged with $\mathrm{La}^{3+}$ ions, in agreement with the results of a previous investigation [20]. On the basis of the mass balance determined by the elemental analysis data, the low atomic 
$(3 \mathrm{La}+\mathrm{Na}) / \mathrm{Al}$ ratio of 0.94 is attributed to possible incidental hydronium ion exchange with the $\mathrm{NaY}$ zeolite and, therefore, 3.2 protons are included in our representation of the unit cell composition of the LaNaY zeolite sample.

The micropore volume of the parent $\mathrm{NaY}$ zeolite determined by liquid $\mathrm{N}_{2}$ adsorption was found to be $0.326 \mathrm{~mL} / \mathrm{g}$, confirming the high degree of crystallinity of the sample. The LaNaY zeolite sample had a micropore volume of $0.084 \mathrm{~mL} / \mathrm{g}$. We emphasize that the micropore volume is reported here per unit mass of the respective zeolite samples although the density of the zeolite changed as a consequence of the ion exchange. After three $\mathrm{Na}^{+}$ions (atomic weight: 22.99 $\mathrm{g} / \mathrm{mol}$ ) are replaced by one $\mathrm{La}^{3+}$ ion (atomic weight: $138.91 \mathrm{~g} / \mathrm{mol}$ ). The mass of the unit cell (or the density of the zeolite) increased by $\sim 7.6 \%$ (relative to that of NaY zeolite) when $65 \%$ of the $\mathrm{Na}^{+}$ions were replaced by $\mathrm{La}^{3+}$. Accordingly, $0.084 \mathrm{~mL} / \mathrm{g}$ of micropore volume of LaNaY zeolite as reported here is equivalent to $0.090 \mathrm{~mL} / \mathrm{g}$ on the basis of the initial NaY zeolite; thus, the contribution of the density change to the change in the reported micropore volume is small relative to the substantial change resulting from the addition of the $\mathrm{La}^{3+}$ ions to the intracrystalline space.

Our high-dose $\left(\sim 10^{5}-10^{8} \mathrm{e}^{-} / \mathrm{A}^{2}\right)$ electron microscopy imaging technique [17] was chosen to maximize the signal-to-noise ratios of the images; consequently, the image simulations that have been applied previously with relatively low-dose $\left(<10^{3} \mathrm{e}^{-} / \mathrm{A}^{2}\right)$ techniques were not required. Fig. 2 is a HAADF-STEM image clearly showing individual (isolated) La cations in the zeolite structure along with lanthanum pair sites. Image smoothing was applied for better visualization of the La cations. We analyzed multiple images representing approximately 400 La cations in 
total, finding that the ratio of single, isolated La cations to La cations in pair sites was approximately 4:1. The images give no evidence of groupings of three or more La cations.

The aforementioned images were obtained quickly, before the occurrence of any observable modifications of the zeolite framework under the influence of the electron beam. To elucidate the effect of the electron beam, consecutive images were recorded at the same location of the sample for a period of $180 \mathrm{~s}$, as shown in Fig. 3. The series of images demonstrates the creation of amorphous regions in the sample under the influence of the electron beam, but without any evidence of aggregation of the isolated La cations to form clusters.

The images were analyzed to determine the distances between the La cations in each pair site. Fig. 4b shows an example illustrating the measurement of the La-La distance for the encircled pair of La cations shown in Fig. 4a. The distance between the two peaks based on the intensity profile is reported as the La-La distance. An analysis of all the images indicative of pair

sites indicates a range of 1.44-3.84 $\AA$, with most falling between 2.70 and $2.80 \AA$ (13 of the 42 La pair sites, or $31 \%$ ), as shown in Fig. 4c. The range of these distances in the planar images is attributed to the fact that the two La cations in each pair site were observed at different angles with respect to the zeolite surface.

\section{Discussion}

XPS data have been interpreted as evidence of the presence of lanthanum pair sites in the zeolite [11], presumably bridged by hydroxyl groups, but results of neutron diffraction experiments [15] have been interpreted as evidence of only isolated $\mathrm{La}^{3+}$ ions. Our results confirm the presence of the pair sites, but they show that only a fraction of the lanthanum cations were present in these sites. 
We infer that the La cations in the pair sites separated at a distance of $3.84 \AA$ represent La-La pairs in essentially the same plane and suggest that this is a nearly true interatomic distance. In contrast, we infer that the shorter La-La distances represent pairs in which the two La cations were present at different depths and that these distances are less than the true interatomic distance. Thus, we interpret the relatively short distances (in the range of 2.70-2.80 $\AA$, with an average of $2.75 \pm 0.04 \AA$ ) characterizing 13 of the 42 La pair sites as representing pair sites tilted at an angle of about $45^{\circ}$ with the respect to the zeolite surface (we recognize that the statement is rough, in part because the zeolite surface is not flat). Details of how these calculations were done are presented elsewhere [21].

The La-La distance of $3.84 \AA$ representing La-La pairs is markedly less than the shortest distance between nearby $\mathrm{Al}$ atoms in the faujasite (assuming one $\mathrm{Si}$ atom between two $\mathrm{Al}$ atoms, consistent with Löwenstein's rule), which the crystallographic data characterizing the faujasite determine to be $4.31 \AA$, corresponding to $\mathrm{Al}$ atoms in a 4-ring - it is the diagonal of the 4-ring. Because the extra-framework cations are coordinated by $\mathrm{O}-\mathrm{O}$ distances are more relevant than the $\mathrm{Al}-\mathrm{Al}$ distances. We ignore the shortest $\mathrm{O}-\mathrm{O}$ distances of about $2.63 \AA$ for $\mathrm{O}$ atoms associated with one $\mathrm{T}$ atom and instead consider the $\mathrm{O}-\mathrm{O}$ pairs that have two $\mathrm{T}$ atoms between them (one $\mathrm{Si}$ and one $\mathrm{Al}$ in the case of the lowest possible $\mathrm{Si} / \mathrm{Al}$ atomic ratio in accord with Löwenstein's rule). We know the following distances on the basis of crystallographic data (restricted here to a maximum of $4 \AA$ ): (1) if they are in 4-rings (different 4-rings): $\mathrm{O}-\mathrm{O}=3.36 \AA$ (O2-O2), $3.58 \AA(\mathrm{O} 4-\mathrm{O} 4), 3.59 \AA(\mathrm{O} 1-\mathrm{O} 1)$, or $3.66 \AA(\mathrm{O} 3-\mathrm{O} 2)$; (2) if they are in a 6-ring: O-O $=3.76 \AA(\mathrm{O} 2-\mathrm{O} 2)$ or $3.80 \AA(\mathrm{O} 3-\mathrm{O} 3)$; and (3) if they span different rings: $\mathrm{O}-\mathrm{O}=4.00 \AA(\mathrm{O} 2-$ $\mathrm{O} 2$ ) (one $\mathrm{O}$ atom in one 4-ring and the other $\mathrm{O}$ atom in the neighboring 4-ring). Because the observed La-La distance in the pair sites $(3.84 \AA)$ is close to these $\mathrm{O}-\mathrm{O}$ distances, we might 
suggest that the lanthanum pair sites represent isolated La cations located nearby each other. However, these $\mathrm{O}-\mathrm{O}$ distances are based on the FAU structure with a Si/Al atomic ratio of 1 [22] whereas the $\mathrm{Si} / \mathrm{Al}$ atomic ratio in our FAU sample was about 2.5 , implying that the $\mathrm{O}-\mathrm{O}$ distances associated with $\mathrm{Al}$ sites in our sample are longer than the La-La distance of $3.84 \AA$. Thus, we infer, these La-La pairs more likely represent dimer-like structures with pairs of La cations probably bridged with one or two $\mathrm{O}$ anions. This interpretation is consistent with the distance between two La cations in $\mathrm{La}_{2} \mathrm{O}_{3}(\sim 3.88 \AA)$ — and with the La-O-La dimer-like structures found for lanthanum dispersed on other supports [23,24].

The data raise the question of why only about $20 \%$ of the La cations were present in pair sites and the others site isolated. On the basis of the analysis of the effects of the electron beam on the samples, we discount the possibility that the beam causes the formation of pair sites. We cannot however rule out the possibility that the isolated and paired La cations were present in different crystallographic locations within the zeolite structure.

The images presented here indicate further opportunities for the application of STEM for the characterization of rare earth ions in zeolites, but much less success is to be expected with the light yttrium than the heavy lanthanum. The method may be of value for characterizing the aging, deactivation, and hydrothermal treatment during regeneration of cracking catalysts and for characterizing the movement of heavy metal cations in such important industrial catalysts. STEM images have already been reported for gold and iridium atoms in zeolites [16-18], and further success is expected for other heavy metals such as platinum.

\section{Conclusions}

In summary, our results demonstrate that aberration-corrected STEM imaging is valuable for visualizing La cations in zeolite $\mathrm{Y}$ with atomic resolution. The results confirm earlier inferences 
based on X-ray photoelectron spectroscopy of the presence of lanthanum pair sites in zeolite Y.

The results are interpreted as evidence that the lanthanum cations in pair sites are present in dimeric structures that are likely bridged with $\mathrm{O}$ anions. STEM offers excellent opportunities for developing new fundamental understanding of heavy metals in zeolites.

\section{Acknowledgments}

We thank Dr. Dan Xie of Chevron for helpful comments. This work was supported by the U.S. Department of Energy (DOE), Office of Science, Basic Energy Sciences, Grants DE-FG0204ER15513 (J.L.), DE-FG02-03ER46057 (P.X., C.A.), and DE-SC0005822 (L.D.) through the University of California, Davis, and the Laboratory Directed Research and Development Program: Chemical Imaging Initiative at Pacific Northwest National Laboratory (PNNL), and the Environmental Molecular Sciences Laboratory, a national scientific user facility sponsored by the DOE Office of Biological and Environmental Research and located at PNNL, a multiprogram national laboratory operated by Battelle for DOE under Contract DE-AC05-76RL01830. P.X. was partially supported by the China Scholarship Council doctoral fellowship program.

\section{References}

[1] W. Vermeiren, J.P. Gilson, Top. Catal. 52 (2009) 1131.

[2] C. S. Hsu, P. R. Robinson, Practical Advances in Petroleum Processing, vol. 1. Springer, New York, 2006. Chapter 3.

[3] C. Martinez, A. Corma, Coord. Chem. Rev. 255 (2011) 1558.

[4] T.F. Degnan, Top. Catal. 13 (2000) 349.

[5] D. Keir, E.F.T. Lee, L.V.C. Rees, Zeolites 8 (1988) 228.

[6] R. Carvajal, P.J. Chu, J.H. Lunsford, J. Catal. 125 (1990) 123.

[7] K. Gaare, D. Akporiaye, J. Mol. Catal. A: Chem. 109 (1996) 177.

[8] Y. Zhang, C. Xiong, Catal. Sci. Technol. 2 (2012) 606.

[9] S. Xiao, R. Le Van Mao, G. Denes, J. Matl. Chem. 5 (1995) 1251.

[10] F. Schüßler, E.A. Pidko, R. Kolvenbach, C. Sievers, E.J.M. Hensen, R.A. van Santen, J.A. Lercher, J. Phys. Chem. C 115 (2011) 21763.

[11] W. Grünert, U. Sauerlandt, R. Schlögl, H.G. Karge, J. Phys. Chem., 97 (1993) 1413. 
[12] J.V. Smith, J.M. Bennett, E.M. Flanigen, Nature 215 (1967) 241.

[13] E.F.T. Lee, L.V.C. Rees, Zeolites 7 (1987) 143.

[14] H. Klein, H. Füss, M. Hunger, J. Chem. Soc., Faraday Trans. 91 (1995) 1813.

[15] A.K. Cheetham, M.M. Eddy, J.M. Thomas, J. Chem. Soc. Chem. Commun. (1984) 1337.

[16] J. Lu, C. Aydin, N.D. Browning, B.C. Gates, Angew. Chem. Int. Ed. 51 (2012) 5842.

[17] C. Aydin, J. Lu, M. Shirai, N.D. Browning, B.C. Gates, ACS Catal. 1 (2011) 1613.

[18] V. Ortalan, A. Uzun, B.C. Gates, N.D. Browning, Nat. Nanotechnol. 5 (2010) 506.

[19] S. Rabl, A. Haas, D. Santi, C. Flego, M. Ferrari, V. Calemma, J. Weitkamp, Appl. Catal. A:

Gen. 400 (2011) 131.

[20] H.S. Sherry, J. Phys. Chem. 70 (1966) 1158.

[21] A. Kulkarni, M.F. Chi, V. Ortalan, N.D. Browning, B.C. Gates, Angew. Chem. Int. Ed. 49 (2010) 10089.

[22] http://izasc-mirror.la.asu.edu/fmi/xsl/IZA-SC/ftc_fw.xsl?-db=Atlas_main\&-lay=fw\&max $=25 \&$ STC $=$ FAU $\&$-find (February 23, 2015).

[23] G.A.H. Mekhemer, Phys. Chem. Chem. Phys. 4 (2002) 5400.

[24] O.V. Manoilova, S.G. Podkolzin, B. Tope, J. Lercher, E.E. Stangland, J.M. Goupil, B.M.

Weckhuysen, J. Phys. Chem. B 108 (2004) 15770. 
Figures

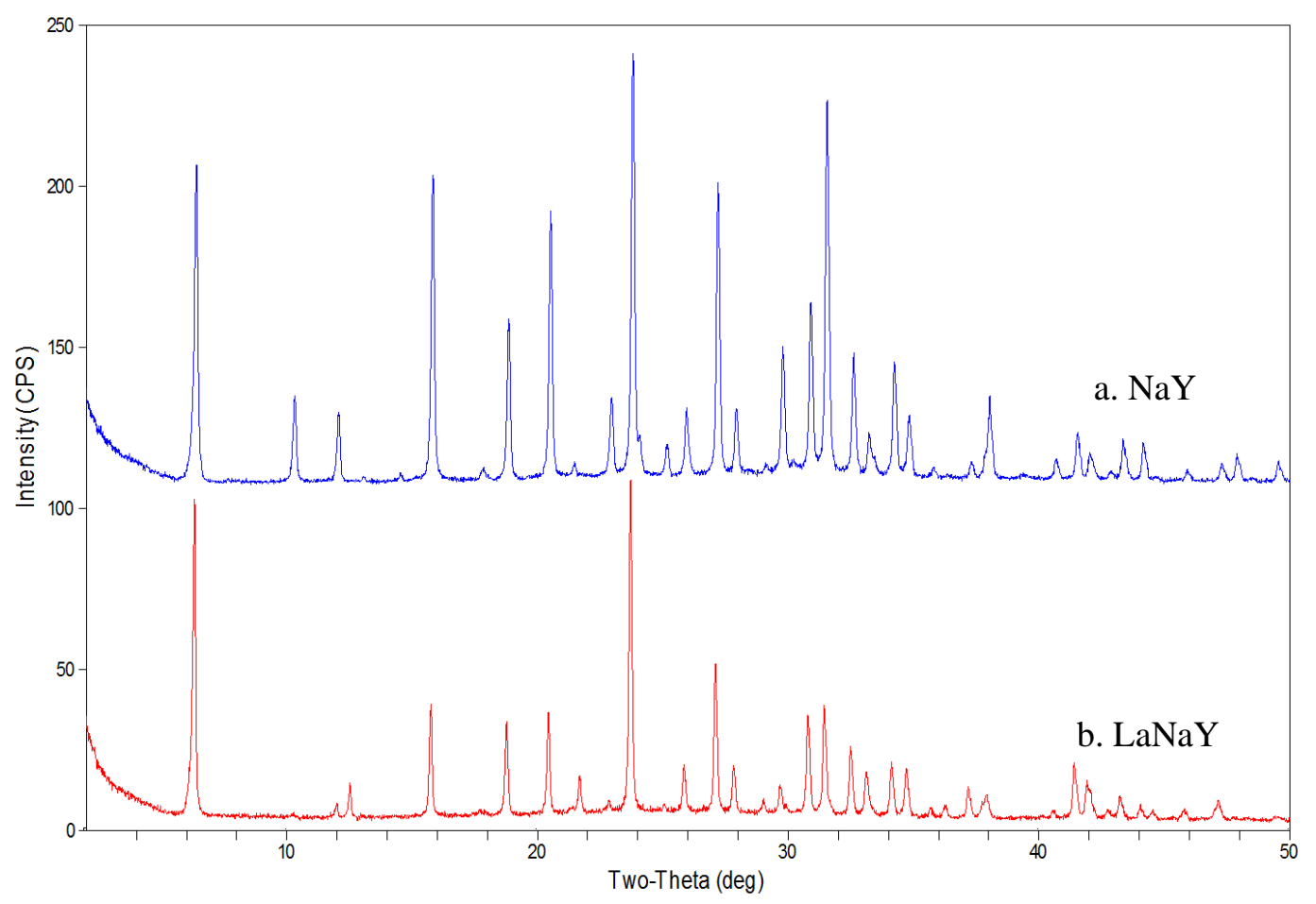

Fig. 1. X-ray diffraction data characterizing (a) the parent $\mathrm{NaY}$ zeolite and (b) LaNaY zeolite. 


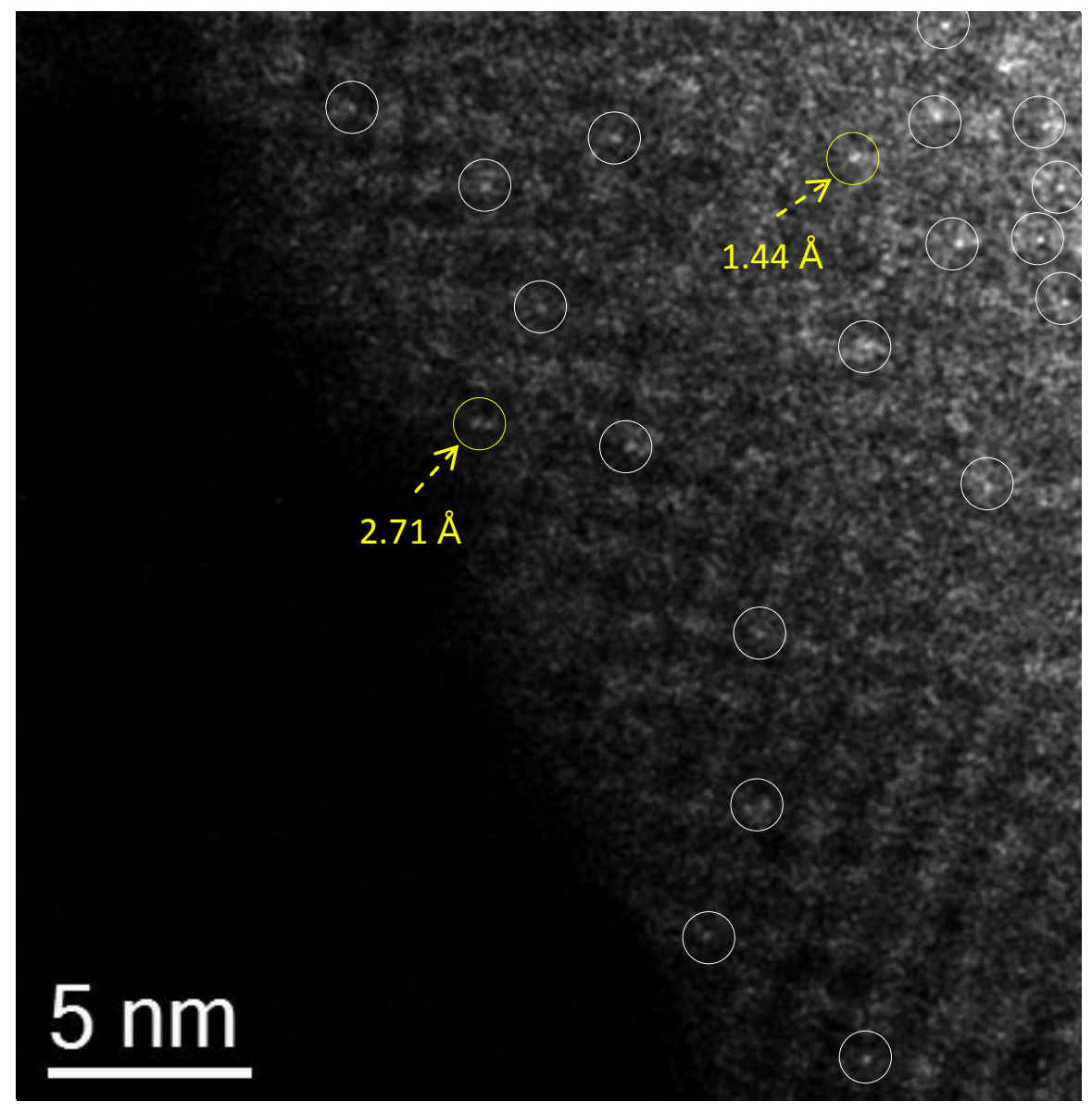

Fig. 2. Aberration-corrected HADDF-STEM image showing both single, isolated La cations and La cations in pairs supported on zeolite Y. Encircled in white are the site-isolated La cations and in yellow the La cation pairs. The distances between La cations in pairs as directly measured on this image are shown. 

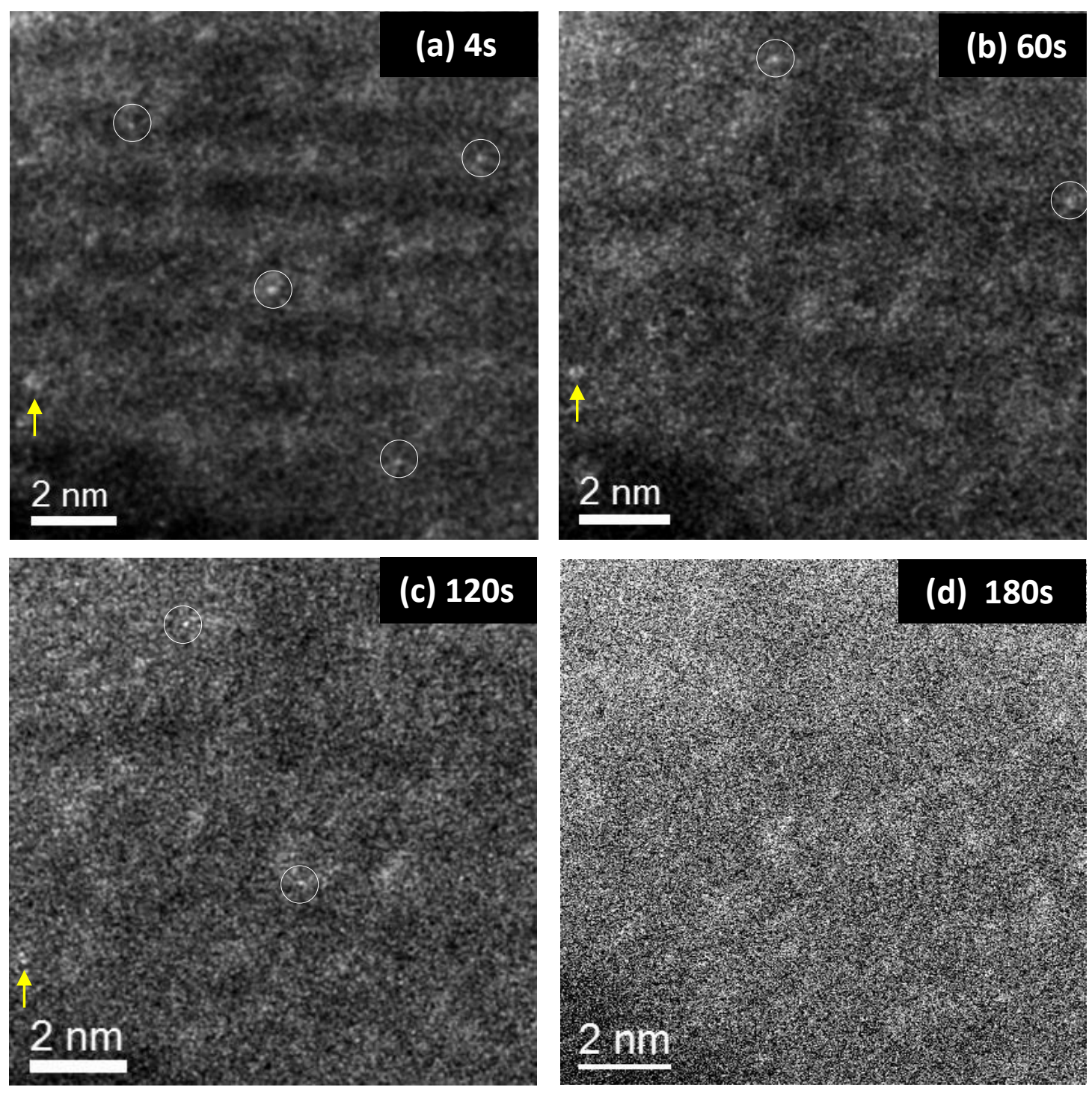

Fig. 3. Consecutive HADDF-STEM images recorded in the same region of the LaNaY zeolite sample for a period of $180 \mathrm{~s}$ showing the absence of lanthanum aggregation to form clusters under the influence of the electron beam. Lanthanum cations are encircled in white, and lanthanum pair sites are indicated by yellow arrows. 

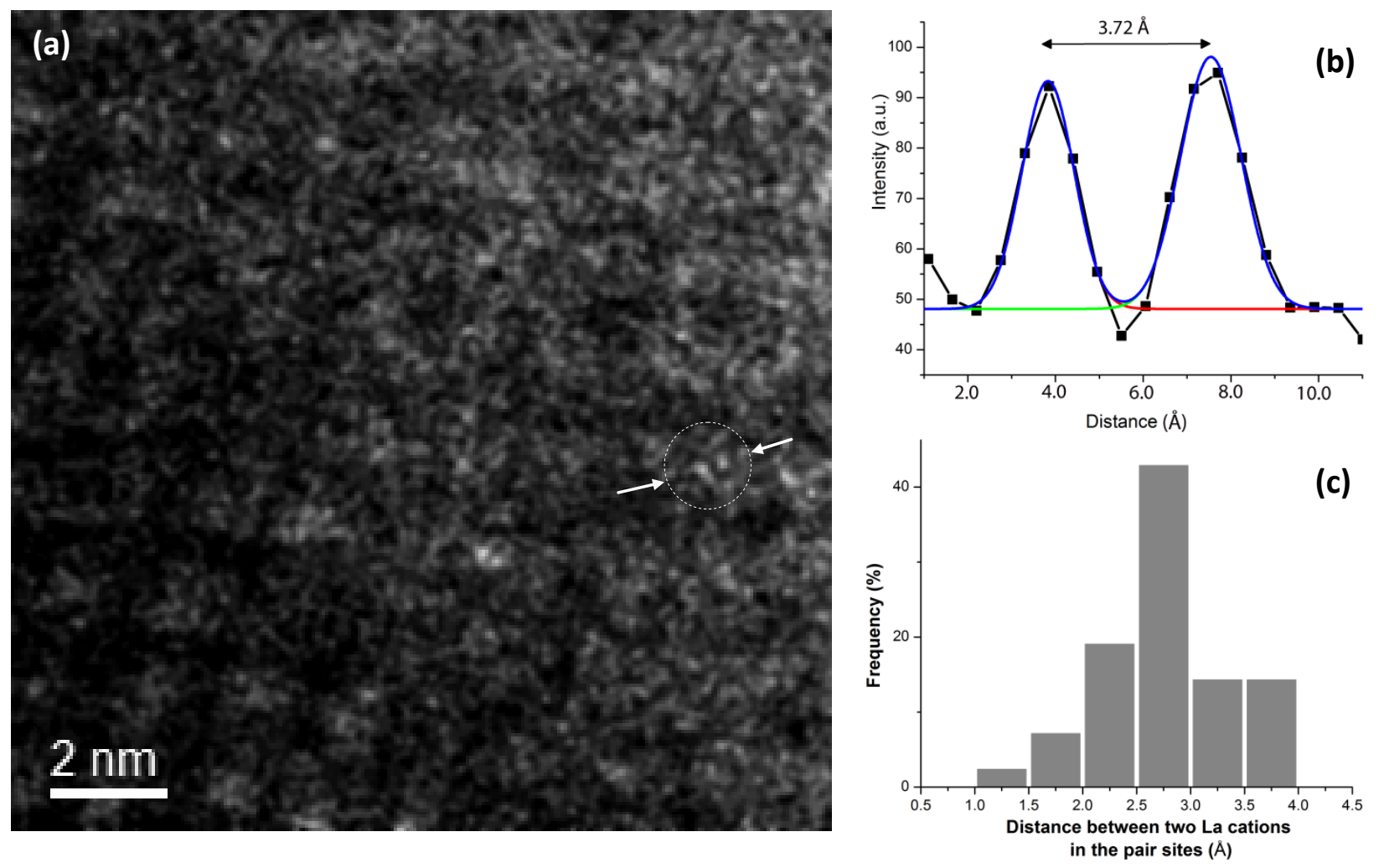

Fig. 4. (a) High-resolution HAADF-STEM image showing an example of a La pair site encircled in white, with a line drawn for determination of an intensity line profile to calculate the distance between two La cations in the pair. (b) Intensity line profile (black) created using Digital Micrograph (Gatan), and characterized by a Gaussian fit (blue) using OriginPro characterizing the example pair in (a). The difference between the two peaks based on the intensity profile is reported as the La-La distance. (c) Histogram illustrating the distribution of La-La distances in 42 pair sites. 